\title{
A control strategy for combined series-parallel pumps
}

\author{
Jose-M. Bueno-Barrachina ${ }^{1}$, César-S. Cañas-Peñuelas ${ }^{1}$, S. Catalan-Izquierdo ${ }^{1}$. \\ 1. Instituto de Tecnología Eléctrica \\ Universidad Politécnica de Valencia \\ Edificio 5E, Tercera Planta \\ Camino de Vera s/n 46022 Valencia (España) \\ Phone/Fax number: +34 96 3877593, +34 963877599 \\ E-mail: jmanuel.bueno@ite.es, cscanas@ite.upv.es, scatalan@ite.upv.es
}

\begin{abstract}
.
When a solution is adopted for a pumping irrigation system, to variable or constant wealth, the engineer has to evaluate the different alternatives to decide the most suitable system of pumping. The majority of the companies of the sector, they choose for systems of pump gradation in parallel at constant speed, with what the variable that they control is the wealth. In this paper there is proposed a system of pumping that connects pumps in parallel and pumps in series, working at variable speed, controlled by an algorithm that allows that every bomb should work inside the area of maximum efficiency.
\end{abstract}

\section{Key words}

Control strategies, Energy efficiency, Environmental Sustainability

\section{Introduction}

Due to the scarcity of the water resources, the majority of the irrigation communities are incorporating systems of pumping that extract the water of underground aquifers and send it to irrigation rafts to a certain level. With these systems they manage to assure the supply of irrigation for his farms.

\section{A Irrigation Raft}

In case of a located irrigation community, that is to say, with only one irrigation raft, the most popular technological solution is to install a pumping station, provided with several centrifugal pumps in parallel [1]. The calculation of the pump-groups is realized for a few certain points of functioning and the system of control is formed so that these points of functioning are reached staggering the starting of the pump-groups and changing the grade of opening of the control valves.

At present they are already used variadores of frequency (ASDs - Adjustable speed drivers) to be able to change the speed of the pump-groups and this way improve the efficiency of the pumping station.

\section{Several Irrigation Rafts}

The problem of these systems arises when it is necessary to give water to two or more irrigation rafts, with different functioning points and separated several kilometers.

Due to the high costs that the civil work represents at the stations of pumping, the most stated thing is to try to satisfy the demand from only one station of pumping, by means of the use of a custom control system that manages the grade of opening of several electrical actuated valves, to satisfy the demands points that the irrigation community. This solution presents two disadvantages:

- The valves in the hydraulic systems are equivalent to losses, for what they reduce efficiency to the set

- In many cases the demands points are unattainables.

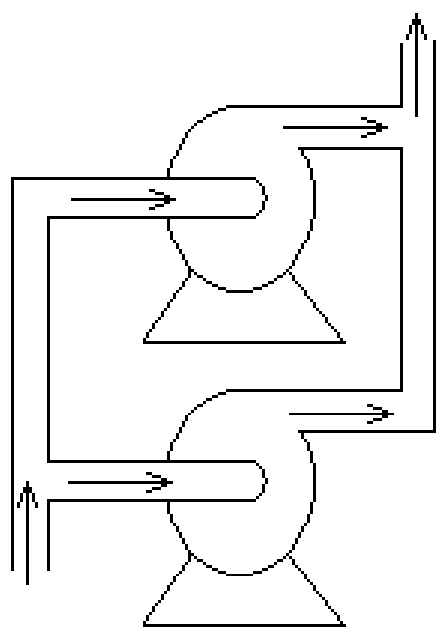

Figure 1: Parallel pumps association 


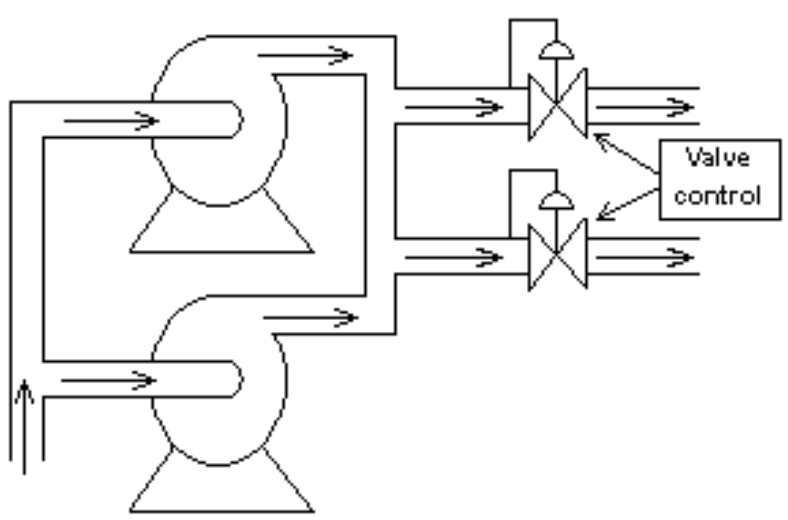

Figure 2: Parallel pumps association with valve control

In the case, in which with only one pumping station there, even installing a custom control system of electrical actuated valves [2], cannot be reached the points of demanded functioning, the conventional solution that is applied at present, it is to install two stations of pumping, treating the irrigation rafts as independent systems. The problem of this solution, it is the high cost of the infrastructure, since in multitude of occasions it is necessary to replicate the pump-groups installed, that generally they will not work to $100 \%$, except exceptional cases.

In this paper we are going to contribute a solution to this case, but using only one station of pumping with this premise, propose the affiliation of pump-groups in parallel with pump-groups in series, with the but to replace the electrical actuated valves control, which introduces losses in the circuit, for a control of starter and speed regulation of the pump-groups that exists at the station of pumping [3,4]. This system of control is in charge of adapting the points of functioning of the pumps to reach the points of functioning demanded with the biggest possible efficiency of the system.

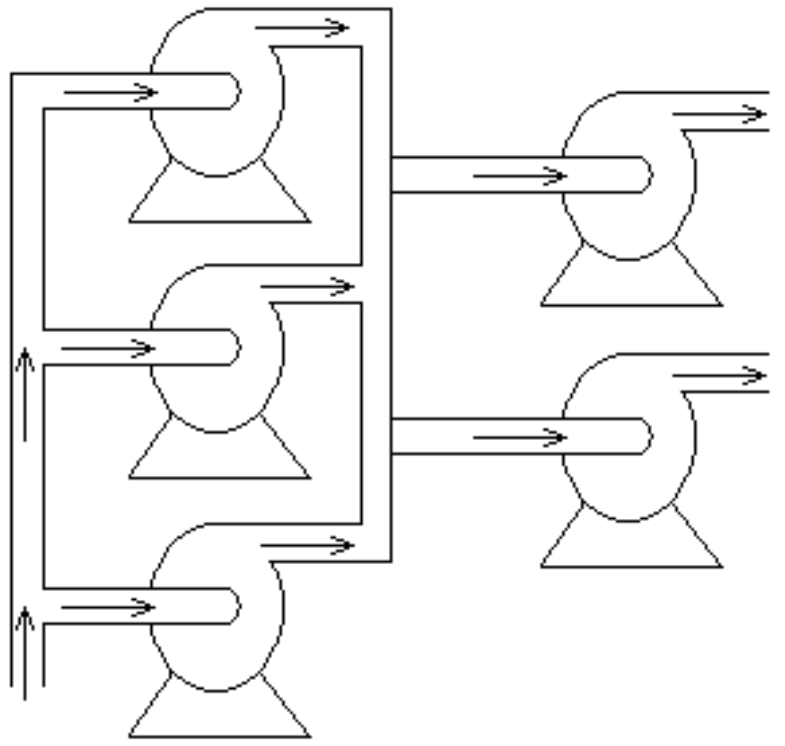

Figure 3: Serial - Parallel pumps association

\section{Control Algorithm}

For this particular case, an algorithm of control has been chosen in closed loop, which manages both the pressure and the wealth delivered by the system, thus he assures that they should not exist overpressures in the canalizations, minimizing the losses for conduction and reducing the costs of maintenance.

Also the system of control is capable of reaching all the demand points indicated by the users, with the biggest energy efficiency possible.

\subsection{System Equations}

To facilitate the algorithm comprehension, we present the application of the optimization algorithm for the case of two non-equals parallel pumps, with the only measurement of the exit pressure of the pumping station.

Applying the pumps scaling laws [5], it is known that the pump behavior equations depend on his draft speed, these relations come given by the following expressions:

$$
\begin{aligned}
& H_{i}=A_{i} \cdot Q_{i}^{2}+B_{i} \cdot \alpha_{i} \cdot Q_{i}+C_{i} \cdot \alpha_{i}^{2} \\
& \eta_{i}=\frac{F_{i}}{\alpha_{i}^{2}} \cdot Q_{i}^{2}+\frac{E_{i}}{\alpha_{i}} \cdot Q_{i} \\
& \text { Nelec }_{i}=\frac{\gamma \cdot Q_{i} \cdot H_{i}}{\eta_{i} \cdot \eta_{\text {electr }_{i}}}
\end{aligned}
$$

Where, for every i-pump:

$H_{i}$ it is the pressure that contributes the pump (m).

$Q_{i}$ it is the wealth of the pump exit $\left(\mathrm{m}^{3} / \mathrm{h}\right)$.

$\alpha_{i}$ it is the draft Speedy of the pump (rpm).

$A_{i}, B_{i}, C_{i}, E_{i}, F_{i}$ there are the coefficients that characterize the pump (information of the manufacturer for a certain operation point).

$\eta_{i}$ it is the efficiency of pump.

$\eta_{\text {eleci }}$ it is the electrical efficiency of a pumpgroup.

Nelec $_{i}$ it is the electrical consumption (kW).

It is necessary to bear in mind that the coefficients $A_{i}, B_{i}, C_{i}, E_{i}, F_{i}$, they are valid for the proximities of the operation point given by the manufacturer, for what if we make the bomb work far from this operation point, the described coefficients can change.

In figure 4, there appears the frequency dependency of the equations (1) and (2). Seeing this figure it is possible to conclude, that changing the frequency, it is possible to make to be employed to the system at another operation point, and that also his yield also will change. 

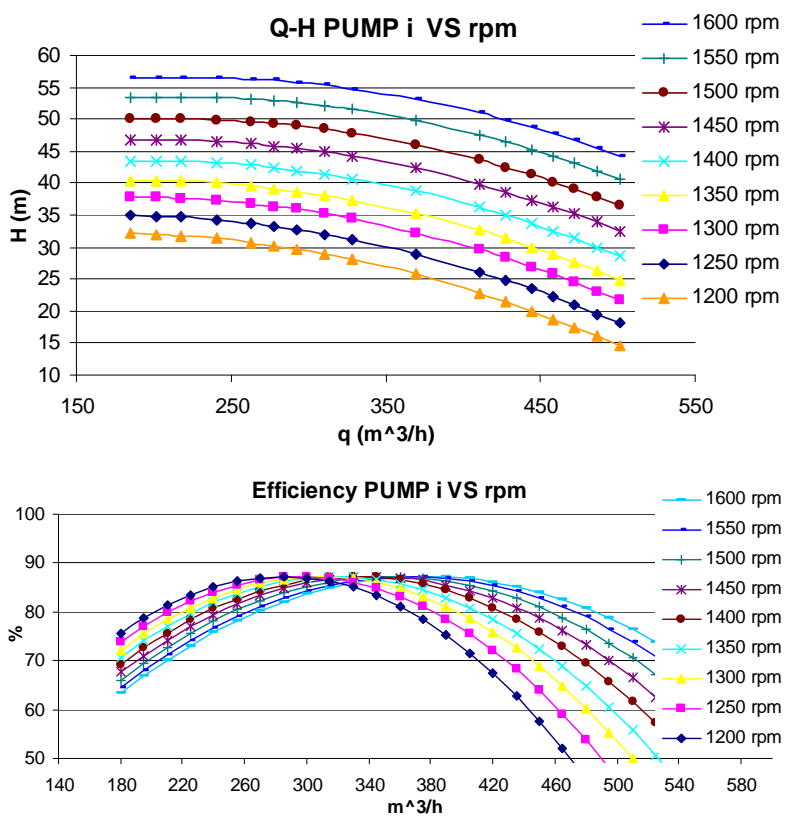

Figure 4: Serial - Characteristic curves of hydraulic pump

Another conclusion that is obtained of figure 4, the fact is that if we fix a certain pressure of exit and change the draft speed, also it will change the wealth of exit and the efficiency. With this result, it is automatic to conclude that for every pressure, there exists a wealth dependent on the draft speed, which maximizes the efficiency of the pump-group, which is equivalent to minimize the electrical consumption.

With this result, it is possible to tackle any pump grouping, like a classical optimization problem.

In case of parallel pump-grouping, it is necessary to bear in mind the following equations, which characterize this type of grouping.

$$
\begin{aligned}
& H_{T}=H_{i}=H_{j} \\
& Q_{T}=Q_{i}+Q_{j}
\end{aligned}
$$

For the case of series-grouping the typical equations will be:

$$
\begin{aligned}
& Q_{T}=Q_{i}=Q_{j} \\
& H_{T}=H_{i}+H_{j}
\end{aligned}
$$

Where, the coefficients " $i, j$ " denote the different pumps that form the grouping.

Of widespread form, the target function of this type of problems comes given by the following equation:

$$
\text { MIN : } N_{\text {electr }_{-} T}=N_{\text {electr_ } i}+N_{\text {electr }_{-} j}
$$

If we concentrate on the equation of the electrical consumption of the pumping group (3), we see that the variables, of the system they do not allow to apply directly the algorithms of linear programming conventional (LPs) (simplex algorihm of Dantzing, ellipsoid algorithm-following Khachiyan-, interior points methods-following Karmarkar-, ...), but it is necessary to use optimization algorithms based on the nonlinear programming (NLP), the first way of tackling these problems, is to uses special formulations of linear programming problems (Wolfe method). Another method involves the uses of branch and bound $(\mathrm{BB}$ or $\mathrm{B} \& \mathrm{~B})$ techniques, where the program is divided into subclasses to be solved with convex or linear approximations that form to lower bound on the overall cost within the part. With subsequent divisions, at some point a current solution will be obtained whose cost is equal to the best lower bound obtained for any of the approximate solutions.

In this particular case, since the pumps groups have to be employed at a certain revolutions range, a method has used based in $\mathrm{B} \& \mathrm{~B}$, but that contemplates the possibility that the pumps that have to work below the limit of revolutions given, are stopped and that there are other pumps those that take charge of the process.

\subsection{Optimization algorithm}

Next it appears to the blocks diagram of the optimization algorithm that is applied to a system formed by two nonequal parallel pumps.

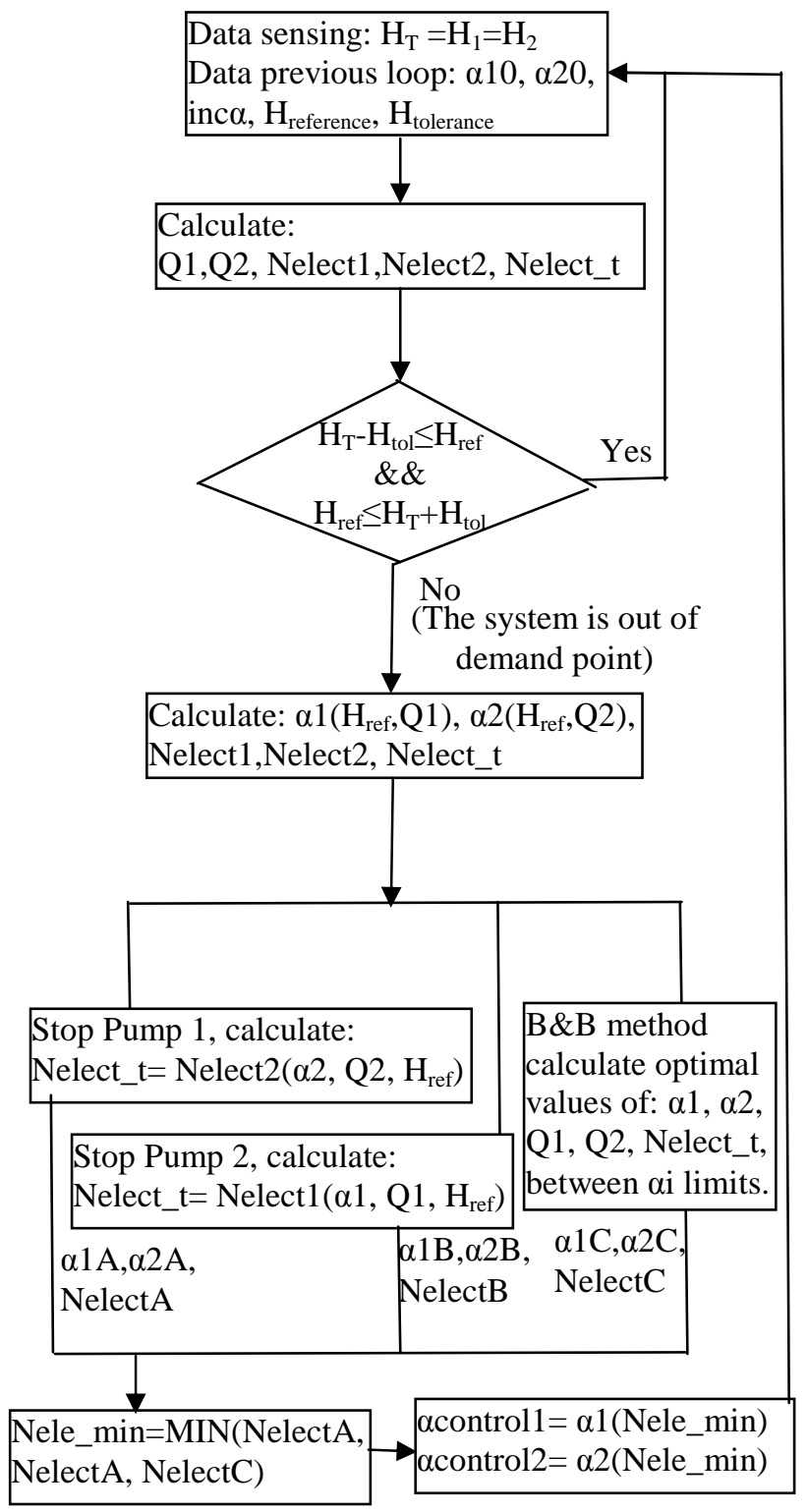


The previous optimization loop, it is designed so that in several executions of the optimization algorithm, the changes in the speeds pumps achieve that the system reaches the working point demanded by the users, assuring an electrical maximum efficiency of the pumping station.

\subsection{Prototipe}

The described control system, it is implemented in a Programmable Automation Controller (PAC), who communicates with the technician of the station of pumping across a touchscreen.

The PAC, it has analogical inputs and outputs, by which it communicates with the exterior.

With some of the analogical outputs of the PAC (4-20 $\mathrm{mA}$ ), there is sent the action of control for the frequency variators that are in charge of controlling the draft speed of the pumps, with the following conversion relation:

$$
4 m A \rightarrow 0 H z
$$$$
20 \mathrm{~mA} \rightarrow 60 \mathrm{~Hz}
$$

By means of some analogical inputs, the system receives information of the different sensors that it has installed. This information serves so that you work the optimized control and to supervise the system in search of mistakes (management of alarms).

The PAC incorporates an additional output, which is a module of RF communication and that allows him to be connected by a supervisor system (SCADA) that fixes the reference pressures and that can stop the entire system or partially in any moment.

In the following figure there appears the blocks diagram of the prototype control system.

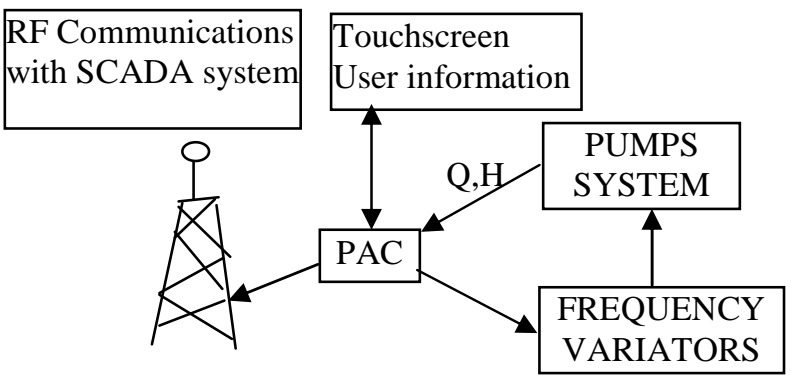

\section{Acknowledgment}

The authors would like to thank Gomaig S.L, for your collaboration in this project, and Ph.D. Yiyao Ye_Lin for her assistance in the optimization algorithm.

\section{Conclusion}

The pumping system proposed is capable of reaching the demand points foreseen by means of the change of the speed of the pump groups installed.

On having chosen for an algorithm of regulation for speed change, also there are changed the efficiency of the system, to make the system to work at the biggest energy efficiency possible.

\section{References}

[1] K. C. Wilson, G. R. Addie, A. Sellgren and R. Clift, Slurry Transport Using Centrifugal Pumps, Springer US, 2006, pp. 190-226.

[2] Rockwell Automation, Ajustable Speed Drives As Applied To Centrifugal Pumps.

[3] Simo Hammo and Juha Viholainen, "Providing flow measurement in parallel pumping systems from variable speed drives", World Pumps, Vol. 2006, Iss. 483.

[4] A. I. Shvindin and A. A. Ivanyushin, "Operation of Centrifugal Pumps in Off-Design Conditions", Chemical and Petroleum Engineering, Vol. 45, Nos. 3-4, 2009.

[5] Mataix Claudio, "Mecánica de Fluidos y Máquinas Hidráulicas", Ediciones del Castillo, $3^{\text {a }}$ Ed. , 1970.

[6] J. Roh; J. -S. Lim; H. -J. Park; J. M. Kang, "An Optimizing Method for Production Systems of Multi-reservoir Gas Fields Using the Branch-system”, Energy Sources, Part A: Recovery, Utilization, and Environmental Effects, Vol. 30, Iss. 1, 2008, pp. 10-19. 